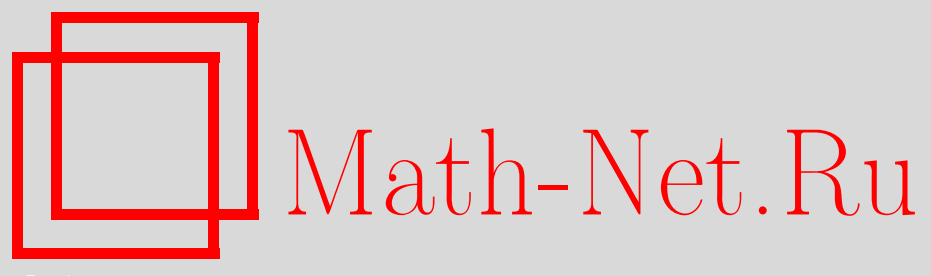

А. В. Комлов, Оценки классов Жеврея данных рассеяния для полиномиальных потенциалов, УМН, 2008, том 63, выпуск 4, 189-190

DOI: https://doi.org/10.4213/rm9220

Использование Общероссийского математического портала Math-Net.Ru подразумевает, что вы прочитали и согласны с пользовательским соглашением http://www . mathnet.ru/rus/agreement

Параметры загрузки:

IP : 35.173 .219 .149

26 апреля 2023 г., 10:00:57

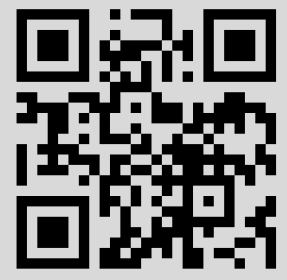




\section{Оценки классов Жеврея данных рассеяния для полиномиальных потенциалов}

\section{А. В. Комлов}

Известно, что необходимым условием разрешимости локальной голоморфной задачи Коши для солитонных уравнений параболического типа является принадлежность данных рассеяния начального условия классу Жеврея $1 / m$, где $m$ - номер потока иерархии (см. [1; теорема 4]). Оказывается, что для мономиальных $(2 \times 2)$-потенциалов удается точно вычислить показатель класса Жеврея их данных рассеяния. В данной работе получена верхняя оценка этого показателя в более общем случае для полиномиальных потенциалов. Она совпадает с нижней оценкой для данных рассеяния мономиальных потенциалов, которая получена в [2] в связи с вопросом о сходимости ряда Концевича-Виттена. (Эта нижняя оценка также может быть получена и методами данной работы.)

Фиксируем произвольную диагональную матрицу $a \in \operatorname{gl}(n, \mathbb{C})$, все собственные значения которой различны. Пусть $q$ - произвольный внедиагональный $\mathrm{gl}(n, \mathbb{C})$-значный $\left(\right.$ т. е. $\left.q_{j j}=0\right)$ голоморфный росток в точке $x_{0} \in \mathbb{C}$. Тогда среди рядов вида $m(x, z)=$ $I+\sum_{s=1}^{\infty} m_{s}(x) z^{-s}$, где $m_{s}(x)-\operatorname{gl}(n, \mathbb{C})$-значный голоморфный росток в точке $x_{0}, c y-$ ществует единственный ряд $m(x, z)$, удовлетворяющий уравнению $m_{x}=[a z, m]+q m$, такой, что все $m_{s}\left(x_{0}\right)$ внедиагональны (см. $\left.[1 ; \S 5]\right)$. Локальными данными рассеяния потенциала $q$ называется формальный степенной ряд $L q(z):=m\left(x_{0}, z\right)-I$.

Формальный степенной ряд $\sum_{s=1}^{\infty} c_{s} z^{-s}\left(c_{s} \in \operatorname{gl}(n, \mathbb{C})\right)$ принадлежит классу Жеврея с показателем $\alpha$ (обозначаемому $\mathrm{Gev}_{\alpha}$ ), если ряд $\sum_{s=0}^{\infty} c_{s}(s !)^{-\alpha} y^{s}$ имеет ненулевой радиус сходимости.

Везде далее $n=2$ (т. е. $q$ есть $(2 \times 2)$-матрица).

УтвеРЖДЕНИЕ. Пусть элементы $q_{12}(x)$ и $q_{21}(x)$ голоморфного внедиагонального $\operatorname{gl}(2, \mathbb{C})$-значного ростка $q(x)$ в точке $x_{0}$ - полиномы степеней $k$ и $l$ соответственно. Тогда Lq принадлежит классу Жеврея с показателем $(k+l) /(k+l+2)$.

ДокАЗАТЕЛЬСТво. Без ограничения общности можно положить $x_{0}=0$. Обозначим элементы $\left(m_{s}\right)_{12}(x)$ и $\left(m_{s}\right)_{21}(x)$ матрицы $m_{s}(x)$ через $u_{s}(x)$ и $v_{s}(x)$ соответственно. Также пусть $b:=a_{11}-a_{22}$. Нам достаточно доказать, что ряды $\sum_{s=1}^{\infty} u_{s}(0) z^{-s}$ и $\sum_{s=1}^{\infty} v_{s}(0) z^{-s}$ принадлежат $\operatorname{Gev}_{(k+l) /(k+l+2)}$. Мы сделаем это для первого из них, а для второго доказательство аналогично.

Из уравнения $m_{x}=[a z, m]+q m$ получаем рекуррентное соотношение $\left[a, m_{s+1}(x)\right]=$ $m_{s}^{\prime}(x)-q(x) m_{s}(x)(s \geqslant 1)$ с начальным условием $\left[a, m_{1}(x)\right]=-q(x)$. Из этого соотношения и условия внедиагональности ряда $m(0, z)$ следует рекуррентное соотношение для $u_{s}(x)$ :

$$
b u_{s+1}(x)=u_{s}^{\prime}(x)-q_{12}(x) \int_{0}^{x} q_{21}(t) u_{s}(t) d t
$$

с начальным условием $b u_{1}(x)=-q_{12}(x)$. Далее считаем $b=1$, так как случай произвольного $b$ отличается от случая $b=1$ умножением $u_{s}(x)$ на $b^{-s}$, что не влияет на принадлежность к классу Жеврея.

Зафиксируем $s$ и оценим $u_{s+1}(0)$. Для этого распишем $u_{s+1}(x)$ по рекуррентной формуле (1) до выражения, зависящего только от $q_{12}(x)$ и $q_{21}(x)$ (применив формулу $s$ раз). Поскольку $q_{12}(x)=\sum_{j=0}^{k} p_{j} x^{j}$ и $q_{21}(x)=\sum_{j=0}^{l} q_{j} x^{j}-$ полиномы степеней $k$ и $l$ соответственно, то, записав их как сумму мономов, получим выражение $u_{s+1}(x)$ через мономы. Всего слагаемых будет не более $2^{s}(k+1)^{s}(l+1)^{s}$. Каждое слагаемое получается из одного из мономов $u_{1}(x)=-q_{12}(x)$ за $s$ шагов, на каждом шаге применяется

Работа выполнена при поддержке РФФИ (грант № 08-01-00014) и программы "Ведущие научные школы" (грант НШ-3877.2008.1). 
одна из двух операций: 1) дифференцирование, 2) интегрирование с умножением берутся два монома $p_{i} x^{i}$ и $q_{j} x^{j}$ из полиномов $q_{12}(x)$ и $q_{21}(x)$ соответственно и $f(x)$ переходит в $-p_{i} q_{j} x^{j} \int_{0}^{x} t^{i} f(t) d t$. Ясно, что на каждом шаге моном переходит в моном.

Оценим модуль коэффициента при каждом из мономов нулевой степени в получившемся выражении для $u_{s+1}(x)$. Заметим, что если на некотором шаге сделано дифференцирование, а на следующем - интегрирование с умножением, то, поменяв операции местами, мы не изменим степени монома, а модуль коэффициента не уменьшится. Поэтому будем считать, что сначала идут все интегрирования с умножением, а затем все дифференцирования. Каждое дифференцирование уменьшает степень монома на 1 , а интегрирование с умножением увеличивает степень монома не более чем на $k+l+1$. Поскольку начальный моном (взятый из $u_{1}(x)=-q_{12}(x)$ ) имеет степень не больше $k$, а в итоге мы должны получить моном степени 0 , то общее число дифференцирований не превосходит $d:=\left[\frac{(k+l+1) s+k}{k+l+2}\right]$, а общее число интегрирований с умножением не меньше $s-d$. Каждое интегрирование с умножением увеличивает степень монома хотя бы на 1, поэтому число, появляющееся при этом в знаменателе, увеличивается на каждом шаге по крайней мере на 1. А после всех дифференцирований должен получиться моном нулевой степени, поэтому за все дифференцирования модуль коэффициента увеличится не более чем в $d$ ! раз. Пусть $M$ - максимум из $1, p_{i}$ и $q_{j}$. Тогда модуль коэффициента монома нулевой степени в выражении для $u_{s}(x)$ не превосходит $M^{2 s+1} d ! /(s-d) !$. Значит,

$$
\left|u_{s+1}(0)\right| \leqslant 2^{s}(k+1)^{s}(l+1)^{s} M^{2 s+1} \frac{d !}{(s-d) !} .
$$

Используя формулу Стирлинга и формулу Коши-Адамара для радиуса сходимости степенного ряда, получаем требуемое утверждение.

Объединяя доказанное утверждение с нижней оценкой, упомянутой в начале работы, получаем следующее предложение.

ПреДЛОЖЕНИЕ. Пусть $k$ u $l$ - целые неотрицательные числа, и пусть элементы $q_{12}(x)$ и $q_{21}(x)$ голоморфного внедиагонального $\mathrm{gl}(2, \mathbb{C})$-значного ростка $q(x)$ в точке $x_{0}$ равны $\alpha\left(x-x_{0}\right)^{k}$ и $\beta\left(x-x_{0}\right)^{l}$ соответственно, где $\alpha$ и $\beta$-ненулевые комплексные числа. Тогда Lq принадлежит классу Жеврея с показателем $(k+l) /(k+l+2)$ и не принадлежит никакому классу Жеврея с меньшим показателем.

\section{Список литературы}

[1] А. В. Домрин, Тр. МИАН, 253 (2006), 46-60. [2] А. В. Домрин, УМН, 63:4 (2008), $185-186$.

\section{А. В. Комлов (А. V. Komlov)}

Московский государственный университет

им. М. В. Ломоносова

E-mail: komlov@hotbox.ru
Представлено А. Г. Сергеевым Принято редколлегией 09.06.2008 